\title{
Adaptive Evolution Strategy SAmple Consensus for 3D Reconstruction from Two Cameras
}

\author{
Yuichiro Toda · Hsu Horng Yz • Takayuki Matsuno • Mamoru Minami . \\ Dalin Zhou
}

Received: date / Accepted: date

\begin{abstract}
RANdom SAmple Consensus (RANSAC) has been applied to many 3D image processing problems such as homography matrix estimation problems and shape detection from 3D point clouds, and is one of the most popular robust estimator methods. However, RANSAC has a problem related to the trade-off between computational cost and stability of search because RANSAC is based on random sampling. In our previous work, we proposed Adaptive Evolution Strategy SAmple Consensus (A-ESSAC) as a new robust estimator, and we applied ESSAC to the homography matrix estimation for 3D SLAM using RGB-D camera. A-ESSAC is based on Evolution Strategy in order to maintain the genetic diversity. Furthermore, ESSAC has two heuristic searches. One is a search range control for reducing the computational cost of RANSAC. The other is adaptive/self-adaptive mutation for changing the search strategy of A-ESSAC according to the best fitness value. In this paper, we apply A-ESSAC to $3 \mathrm{D}$ reconstruction method using two cameras, and we show an experimental result, and discuss the effectiveness of the proposed method.
\end{abstract}

Keywords Evolutionary computation · Robust estimator $\cdot 3 \mathrm{D}$ reconstruction

\section{Introduction}

Recently, 3D image processing technologies have expected as a development of 3D distance measurement sensors such as Lidar and RGB-D camera. These kind

Y. Toda

3-1-1 Tsushima-naka, Kita-ku, Okayama

Tel.: +81-86-251-8924

Fax: +81-86-251-8924

E-mail: ytoda@okayama-u.ac.jp of sensors enable to perform the 3D image processing in real-time and many kinds of applications related with the $3 \mathrm{D}$ image processing have proposed in the fields of robotics and Intelligent Transport Systems. However, the measurement data of the 3D distance measurement sensor includes many noises according to the environments such as the material of the measurement object and the lightning condition. In order to estimate a model from the noisy dataset, RANdom SAmple Consensus (RANSAC) proposed by Fischer and Bolles is one of the most popular algorithms in robust estimator [1]. RANSAC has been applied to many 3D image processing problems such as homography matrix estimation problem and shape detection from the 3D point clouds [2-4]. However, one of the problems in RANSAC is a sampling bias in a search, since it selects candidate pairs from a data set of pairs randomly. In order to solve the problem of RANSAC, many researchers have improved RANSAC algorithm. Choi et al. [5] gave a critical survey of RANSAC family algorithms. They synthesized seven research axes that were: Partial Evaluation (e.g., Progressive RANSAC), Adaptive Termination (e.g., uMLESAC), Adaptive Evaluation (e.g., pbM-estimator), Local Optimization (e.g., LO-RANSAC), Model Selection (e.g., MAPSAC), Loss Function (e.g., MLESAC), and Guided Sampling (e.g., GASAC). These research axes were discussed from different objectives; being fast, being robust, being accurate.

In this paper, our objective is to reconstruct $3 \mathrm{D}$ point cloud from two cameras in real-time from the noisy dataset.Therefore, we focus on evolutionary computation for RANSAC family algorithms because Genetic Algorithm SAmple Consensus (GASAC) proposed by $\mathrm{V}$. Rodehorst and O. Hellwich [6] can improve the performance of the search capability by a population- 
based multi-point search. However, it is sometimes difficult to maintain the genetic diversity in the search if the large size of outliers is included in a data set. In order to solve this problem. E. Shojaedini et al. proposed the modified GASAC as Adaptive Genetic Algorithm Sample Consensus (AGASAC) by applying the adaptive mutation, crossover and learning roulette wheel selection to GASAC algorithm [7]. AGASAC can change the dominant operators (mutation and crossover) according to the fitness values in the gene set. By using these new operators, E. Shojaedini et al. showed AGASAC outperforms GASAC. However, these kinds of evolutionary computation methods require more computing time than any other SAC methods. Therefore, we must deal with the trade-off between computational cost and stability of search.

There are two possible approaches to improve the trade-off. One is to change the strategy of the local search and global search efficiently. This kind of approaches can control the genetic diversity of a population to improve the stability in evolutionary search. The other is to manage the search range in the dataset according to the current search result. This kind of approaches can remove obvious outliers from a dataset. However, the feasible solutions of the model parameters are required to discriminate inliners from outliers. This means that the discrimination of inliners and outliers requires model parameters, while the estimation of model parameters requires a set of inliers. This is a nesting structure each other. In our previous work, we proposed Adaptive Evolution Strategy SAmple Consensus (A-ESSAC) as a new robust estimator method in order to improve the trade-off between computational cost and stability of search in RANSAC. Furtheremore, we applied to A-ESSAC to 3D map building method using RGB-D camera for realizing the real time 3D SLAM [8]. In this paper, we apply A-ESSAC to $3 \mathrm{D}$ reconstruction method using two cameras for verifying the effectiveness and possible application of A-ESSAC. This paper is organized as follows. Section 2 explains our 3D reconstruction method. Section 3 explains A-ESSAC. Section 4 shows experimental results of the proposed method.

\section{$23 \mathrm{D}$ reconstruction using two cameras}

\subsection{Algorithm of 3D reconstruction}

In this paper, we focus on $3 \mathrm{D}$ reconstruction from two cameras. Our 3D reconstruction method uses a local feature extraction method for reconstructing the sparse point cloud data. For the local feature based 3D reconstruction method, the algorithm can mainly divide into two steps. One step is the image processing step that is for searching correct corresponding points between two camera data. The other is the matching step of $3 \mathrm{D}$ point clouds using the relation between two data. In these algorithms, a homography estimation method such as RANSAC is required for extracting the corresponding points. Therefore, we deal with the 3D modeling method as an application of our method. Figure 1 shows the flowchart of this algorithm. In Fig. 1, the $t$-th measurement data from left and right camera are expressed by $D_{L}(t)$ and $D_{R}(t)$, respectively. Specifically, the possible pairs of corresponding points between $D_{L}(t)$ and $D_{R}(t)$ are generated by extracting any features of camera image. However, the pairs include many mismatched pairs when the pairs are generated. Therefore, the homography matrix is estimated by using A-ESSAC (our proposed method) in order to remove the mismatched pairs. Finally, the coordinate transform matrix is estimated by using 3D distance information of the corresponding points in order to update $3 \mathrm{D}$ environmental map.

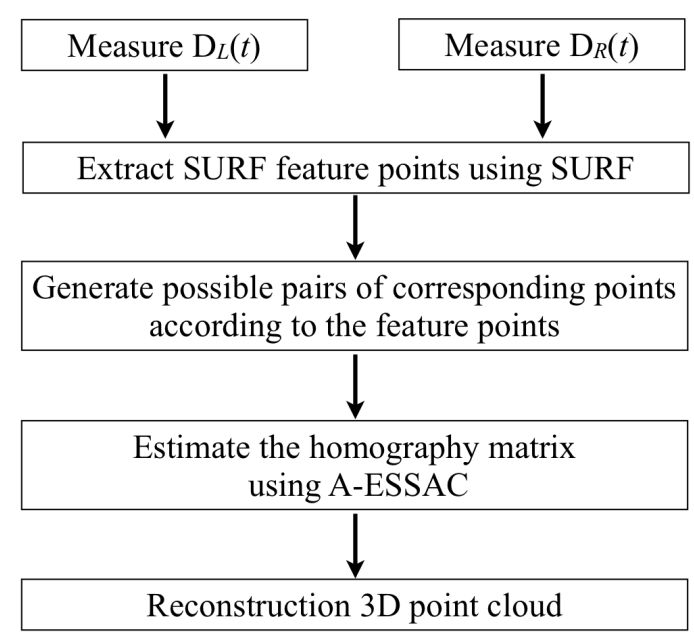

Fig. 1 Flowchart of proposed 3D modeling method.

\subsection{Feature extraction}

This subsection explains the detail of feature extraction and matching method. Recently, vaious types of local features have been proposed for the object recognition and extracting the corresponding points from multiple images. In local features, Scale-Invariant Feature Transform (SIFT) and Speeded Up Robust Features (SURF) are the most commonly used in order to extract the corresponding points from the multiple images $[9,10]$. These local features are robust to the change of illumination and local affine distortion of images. Espe- 
cially, SURF proposed by Bay et al. is based on $2 \mathrm{D}$ Haar wavelet responses as a robust lo-cal feature detector inspired by SIFT. The standard SURF is several times faster than SIFT. Furthermore, we must reduce computational time as much as possible in real-time image processing. General-purpose graphics processing unit (GPGPU) has been applied to calculate and extract features in real-time. In this paper, we use SURF implemented on GPGPU to describe features for pattern matching between the left and right camera images. After extracting SURF from the two images, each SURF feature is described by a vector containing 64 or 128 elements. An initial set for estimating homography matrix (possible pairs of corresponding points) is obtained by selecting the pairs with the minimum Euclidian disrtance of the feature vector between the left and right camera images (Fig. 2).

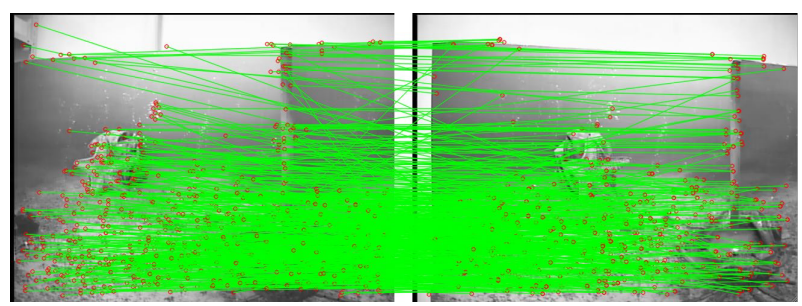

Fig. 2 A result of the feature extraction and matching by using SURF. Red circles and green lines indicate feature points and matching results, respectively.

\subsection{Homography estimation}

After the feature extraction and matching, we should extract the correct pairs of corresponding points from the dataset of possible pairs. In many researches, homography matrix is estimated for extracting the correct pairs $[11,12]$. In this way, the homography matrix estimation problem is one of the most important problems not only in 2D image processing but also in $3 \mathrm{D}$ image processing because the matrix is required in various types of $3 \mathrm{D}$ image processing such as stereovision [12] and 3D environment map building. The homography matrix $\mathrm{H}$ that has 9 elements is the matrix that describes the relation between two images. Fig. 3 displays the concept image of homography matrix between two images. The set of homogeneous image points $\left\{\mathbf{x}_{i}\right\}(i=1, \ldots, n)$ as viewed in the first image is transformed into the set $\mathbf{x}_{\mathbf{i}}^{\prime}$ in the second image, with the positions related by

$\mathbf{x}_{\mathbf{i}}^{\prime}=\mathbf{H x}_{\mathbf{i}}$ where $\mathbf{x}_{\mathbf{i}}$ and $\mathbf{x}_{\mathbf{i}}^{\prime}$ are homogeneous three vectors $\mathbf{x}=$ $(x, y, 1)^{T}, \mathbf{x}^{\prime}=\left(x^{\prime}, y^{\prime}, 1\right)^{T}$ because the matrix $\mathbf{H}$ is composed of $3 \times 3$ matrix. In addition, equation (1) can be also defined by the following equation:

$\begin{aligned} x^{\prime} & =\frac{a_{1} x+a_{2} y+a_{3}}{a_{7} x+a_{8} y+1} \\ y^{\prime} & =\frac{a_{4} x+a_{5} y+a_{6}}{a_{7} x+a_{8} y+1}\end{aligned}$

where $a_{1}-a_{8}$ are the parameters. Therefore, the homography matrix is estimated by estimating these 8 parameters. Estimating the homography matrix between two images enables to extract the correct pairs of corresponding points. However, the set of possible pairs includes many mismatch pairs. Therefore, robust estimators are applied to this problem $[13,14]$.

\subsection{Update of 3D model}

The update of the 3D model is to obtain the position $\mathbf{x}_{r i}^{\prime}=\left(x_{r i}^{\prime}, y_{r i}^{\prime}, z_{r i}^{\prime}\right)$ of a pixel in the 3D space based on the position $\mathbf{x}_{r i}=\left(x_{r i}, y_{r i}, z_{r i}\right)$ according to the relationship between $\left(x_{i}^{\prime}, y_{i}^{\prime}\right)$ and $\left(x_{i}, y_{i}\right)$. An interactive closest point (ICP) algorithm is one of the most widely used methods of matching a set $\left(X^{r}\right)$ of points with point clouds $\left(X_{r}^{\prime}\right)$ in $3 \mathrm{D}$ space [15]. The error function to be minimized is defined as

$E(\mathbf{R}, \mathbf{t})=\frac{1}{N_{c}}\left\|\sum_{i=1}^{N_{c}} \mathbf{R} \mathbf{x}_{r i}^{\prime}+\mathbf{t}-\mathbf{x}_{\mathbf{r i}}\right\|$

where $\mathbf{R}$ is the rotation matrix; $\mathbf{t}$ is the translation vector; We apply the unit quaternion proposed by Horn [16]. The quaternion is defined as $\hat{q}=\left(q_{0}, q_{1}, q_{2}, q_{3}\right)$. First, the center of gravity (COG) of each point cloud is calculated in the following;

$$
\begin{aligned}
\mathbf{x}_{r}^{g} & =\frac{1}{N_{c}} \sum_{i=1}^{N_{c}} \mathbf{x}_{\mathbf{r i}} \\
\mathbf{x}_{r}^{\prime g} & =\frac{1}{N_{c}} \sum_{i=1}^{N_{c}} \mathbf{x}_{\mathbf{r i}}^{\prime}
\end{aligned}
$$

where $N_{c}$ is the number of points in each point cloud. Next, the relative position from the COG is calculated in the following;

$$
\begin{aligned}
\mathrm{x}_{\mathrm{i}}^{\mathrm{a}} & =\mathrm{x}_{\mathrm{ri}}-\mathrm{x}_{\mathrm{r}}^{\mathrm{g}} \\
\mathrm{x}_{\mathrm{i}}^{\prime \mathbf{b}} & =\mathrm{x}_{\mathrm{ri}}^{\prime}-\mathrm{x}_{\mathrm{r}}^{\prime \mathrm{g}}
\end{aligned}
$$

Next, $S_{a b}$ is defined as;

$$
S_{a b}=\sum_{i=1}^{N_{c}} \mathbf{x}_{\mathbf{i}}^{\mathbf{a}} \mathbf{x}_{\mathbf{i}}^{\mathbf{b}}
$$


According to $S_{a b}$, a matrix $\mathbf{P}$ is defined as

$\mathbf{P}=\left(\begin{array}{cccc}S_{x x}+S_{y y}+S_{z z} & S_{y z}-S_{z y} & S_{z x}-S_{x z} & S_{x y}-S_{y x} \\ S_{y z}-S_{z y} & S_{x x}-S_{y y}-S_{z z} & S_{x y}+S_{y x} & S_{z x}+S_{x z} \\ S_{z x}-S_{x z} & S_{x y}+S_{y x} & S_{y y}-S_{x x}-S_{z z} & S_{y z}+S_{z y} \\ S_{x y}-S_{y x} & S_{z x}+S_{x z} & S_{y z}+S_{z y} & S_{z z}-S_{x x}-S_{y y}\end{array}\right)$

Here the eigenvector corresponding to the maximum positive eigenvalue of $\mathbf{P}$ is quaternion $(\hat{q})$. The rotation matrix is obtained by $\hat{q}$ in the following;

$\mathbf{R}=\left(\begin{array}{ccc}q_{0}^{2}+q_{1}^{2}-q_{2}^{2}-q_{3}^{2} & 2\left(q_{1} q_{2}-q_{0} q_{3}\right) & 2\left(q_{1} q_{3}+q_{0} q_{2}\right) \\ 2\left(q_{2} q_{1}+q_{0} q_{3}\right) & q_{0}^{2}-q_{1}^{2}+q_{2}^{2}-q_{3}^{2} & 2\left(q_{2} q_{3}-q_{0} q_{1}\right) \\ 2\left(q_{3} q_{1}-q_{0} q_{2}\right) & 2\left(q_{3} q_{2}+q_{0} q_{1}\right) & q_{0}^{2}-q_{1}^{2}+q_{2}^{2}+q_{3}^{2}\end{array}\right)$

Furthermore, the translation vector is also obtained by $\mathbf{R}$ in the following;

$\mathrm{t}=\mathrm{x}_{\mathrm{r}}^{\mathrm{g}}-\mathbf{R} \mathrm{x}_{\mathrm{ri}}^{\mathrm{g}}$

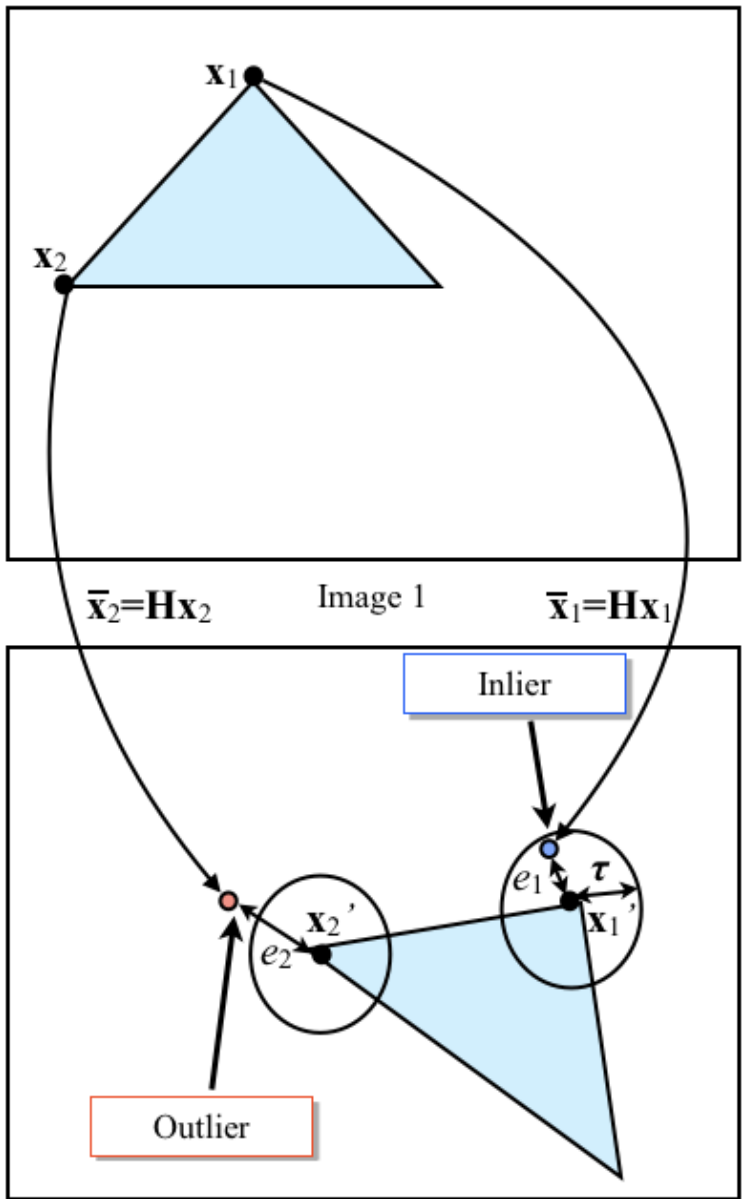

Image 2

Fig. 3 Concept image of 2D homography, where $\overline{\mathbf{x}_{\mathbf{i}}}$ indicates an estimated point of the $i$ th point by using the homography matrix $\mathbf{H}$.

\section{Adaptive Evolution Strategy Sample Consensus (A-ESSAC)}

\subsection{Total algorithm of A-ESSAC}

In ESSAC, the genotype is composed of $k$ candidate data needed to calculate the model parameters and the combination of candidate data is optimized by global search and hill-climbing search using genetic operators. The fitness value $f_{i t}$ is calculated by following fitness function;

fit $_{i}=$ fitnessFunction $(g(i))=\sum_{j} \rho\left(e_{j}\right)$

where $e_{j}$ indicates the $j$ th error calculated by the following equation;

$e_{j}=\left\|\mathbf{x}_{\mathbf{j}}^{\prime}-\mathbf{x}_{\mathbf{j}}\right\|$

where $\mathbf{x}_{\mathbf{j}}$ and $\mathbf{x}_{\mathbf{j}}^{\prime}$ are homogeneous three vectors defined in Eq. (1).

In ESSAC, the fitness function usually uses the number of inliers. Therefore, this problem is a maximization problem. Furthermore, ESSAC has a search range control method in order to reduce computational cost and improve the stability of search simultaneously.

\subsection{Evolution Strategy}

Basically, the random sampling required to estimate parameters of a mathematical model in the generation of hypothesis is one of combinational optimization problems, but we can incorporate local search or heuristics to reduce computational cost. Evolutionary computation (EC) is used to solve optimization problem by simulating evolution on a computer. From the historical point of view, EC can be divided into genetic algorithm (GA), evolutionary programming (EP), and evolution strategy (ES). These methods are fundamentally iterative generation and alternation processes operating on a set of candidate solutions called a population. All the population evolves toward better candidate solutions by selection operation and genetic operators (crossover and mutation). The selection decides candidate solutions evolving into the next generation, which limits the search space spanned by the candidate solutions. The crossover and mutation generate new solution candidates. However, genetic operators used for generating new solution candidates are a little different among GA, EP, and ES from histrorical point of view [17]. The important feature of ES is the self-adaptation which can self-tune the diversity of mutation parameters according to the success records. Rechenberg suggested that the ratio of successful mutations to all mutations should be $1 / 5$ [18]. If this ratio is greater than 
$1 / 5$, increase the variance; if it is less, decrease the variance. This ratio has often been discussed in the previous studies, but the self-adaptive mutation can change the variance of the normal random value according to the success ratio based on the landscape of a fitness function. While a self-adaptive mutation refers to its own fitness record, an adaptive mutation refers to the average, maximum, and minimum of fitness values of the candidate solutions in the population, i.e., the adaptive mutation relatively changes the distribution of genotype in a population according to the fitness values of the candidate solutions. ES was proposed by Rechenberg, and extended further by Schwefel. Basically, ES is classified into $(\mu+\lambda)$-ES and $(\mu, \lambda)$-ES. First, Algorithm 1 presents the procedure of a standard $(\mu+\lambda)$-ES. Initialization randomly generates an initial population of individuals. Creation $(\lambda)$ generates $\lambda$ children from $\mu$ parents by genetic operators in a single generation. As a result, the $(\mu+\lambda)$-ES has the intermediate population of $(\mu+\lambda)$ individuals. Selection $(\mu)$ deterministically selects the best $\mu$ individuals from the intermediate population. On the other hand, in $(\mu, \lambda)$-ES, Selection $(\mu)$ selects the best $\mu$ individuals only from the created $\lambda$ children $(\mu<\lambda)$. Therefore, $(\mu+\lambda)$-ES is considered as a continuous model of generation, while the $(\mu, \lambda)$-ES is considered as a discrete model of generation. Especially, as the special cases of ES, $(1,1)$-ES is a random search, $(1+1)$-ES is an iterative improvement method, $(1, \lambda)$ ES or $(1+\lambda)$-ES is a multi-point neighboring search, and $(\mu+1)$-ES is a local hill-climbing search. In our proposed method, the search method is mainly based on mutation operators and uses self-adaptive mutation since we assume that the dataset includes a huge number of noises. The mutation operator is very important to extract the correct pairs from the dataset effectively. Therefore, we use ES in this study. In ESSAC, we use uniform crossover as a recombination and simple mutation that changes genes randomly according to the mutation rate.

\begin{tabular}{l}
\hline Algorithm 1 Standard ES \\
\hline Initialization \\
while until termination condition is True do \\
Creation $(\lambda)$ \\
$\quad$ Evaluation \\
Selection $(\mu)$ \\
end while \\
\hline
\end{tabular}

\subsection{Search range control}

ESSAC performs a search range control in order to reduce computational cost and improve the stability of search simultaneously. This subsection proposes the search range control method that is the feature of ES$\mathrm{SAC}$. The step that requires computational cost in SAC algorithm is the hypothesis evaluation step. In the hypothesis evaluation, the generated model parameters are evaluated by using all data in a data set $\mathrm{S}$ of the possible pairs. Therefore, if the data set has a huge mount of data such as 3D image processing, the computational cost is very expensive. Furthermore, it is difficult to optimize the combination of candidate data if the outlier rate in the set $S$ is very high. Therefore, in the search range control method of ESSAC, if an individual satisfied with starting condition is generated, the search space is reduced by removing obvious outliers from the data set $S$. Fig. 4 shows the concept image of search range control method of ESSAC. Specifically, by using the model parameters of the best individual and predefined threshold $\tau_{a}\left(\tau_{a}>\tau\right)$ the number of removal data $N_{a}$ and the set $S_{a}$ are calculated as follows,

$$
\begin{aligned}
N_{a} & =\sum_{i=1}^{N} \rho_{i}\left(e_{i}\right) \\
S_{a} \leftarrow & S_{a}+\{i\} \\
\rho_{i}\left(e_{i}\right) & = \begin{cases}1 & \left(\text { if } e_{i}>\tau_{a}\right) \\
0 & \text { (otherwise) }\end{cases}
\end{aligned}
$$

Furthermore, by using $N_{a}$ and $S_{a}$, the number of data $N$ and the set $S$ are updated as follows

$N \leftarrow N-N_{a}$

$S \leftarrow S-S_{a}$

By removing the obvious outliers from the dataset $S$, ESSAC reduces the computational cost by the number of obvious outliers $N_{a}$ in the evaluation step as compared with RANSAC algorithm. Therefore, the computational cost of ESSAC depends on the rate of the outliers in the dataset is significantly reduced as the rate is increased.The model parameters are estimated from the updated data set $S$. In addition, the starting condition in homography matrix estimation are defined by

$\frac{f_{i t_{\text {best }}}}{N}>\alpha$

where $\alpha$ is a threshold value. The condition means the ratio of the fitness value of the best individual to the number of data in the data set. After the search range control method is once performed, the obvious outliers are removed from the set $S$ when the best individual is improved.

\subsection{Adaptive mutation}

This subsection explains the adaptive mutation rate that enables to change the mutation rate according to 
the fitness value of the best individual in order to improve the stability and accuracy for ESSAC. Because if the outlier rate is high, the good combination is not generated even in later phase of the search in some cases. Specifically, the adaptive mutation rate is determined by the following equation

$P_{m 1}=1-\exp \left(-\frac{f i t_{\text {best }}}{T_{m}}\right)$

where fit $_{\text {best }}$ is the fitness value of the best individual and $T_{m}$ is coefficient. In this paper, we use $T_{m}=0.2 \cdot N$. On the other hand, the search is based on the recombination operator in equation (20) when the fitness value of the best individual is low during an early stage. In equation (20), the recombination randomly selects two host individuals in order to maintain the genetic diversity.

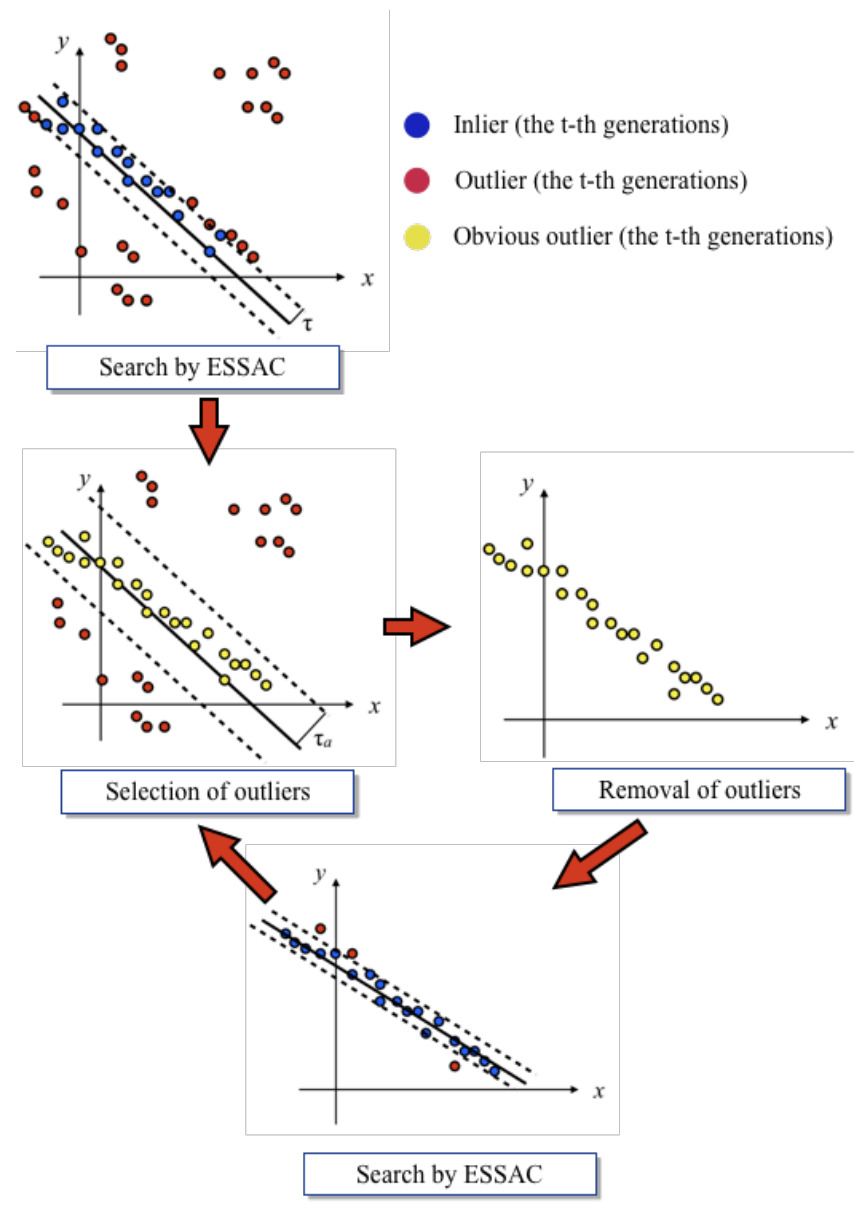

Fig. 4 Concept image of search range control method in ESSAC.

\subsection{Self-adaptive mutation}

In order to start search range control method efficiently, the search capability of ES is very important since we must search the feasible solution from the dataset including the large number of outliers quickly. Therefore, we need to control a ratio of exploration and exploitation. In order to control the ratio, ESSAC uses a selfadaptive mutation. In the adaptive mutation, if the search fails for $\mathrm{m}$ times in a row, the mutation rate is calculated by following equation,

$P_{m 2}=1-P_{m 1}$

where $P_{m 1}$ is calculated by equation (20). In equation (21), the search is based on the mutation operator when the fitness value of the best individual is low and the mutation rate decreases when an individual having good genetic information is generated. Therefore, ESSAC can control the ratio by using adaptive and selfadaptive mutation. Algorithm 2 shows the procedure of adaptive ESSAC.

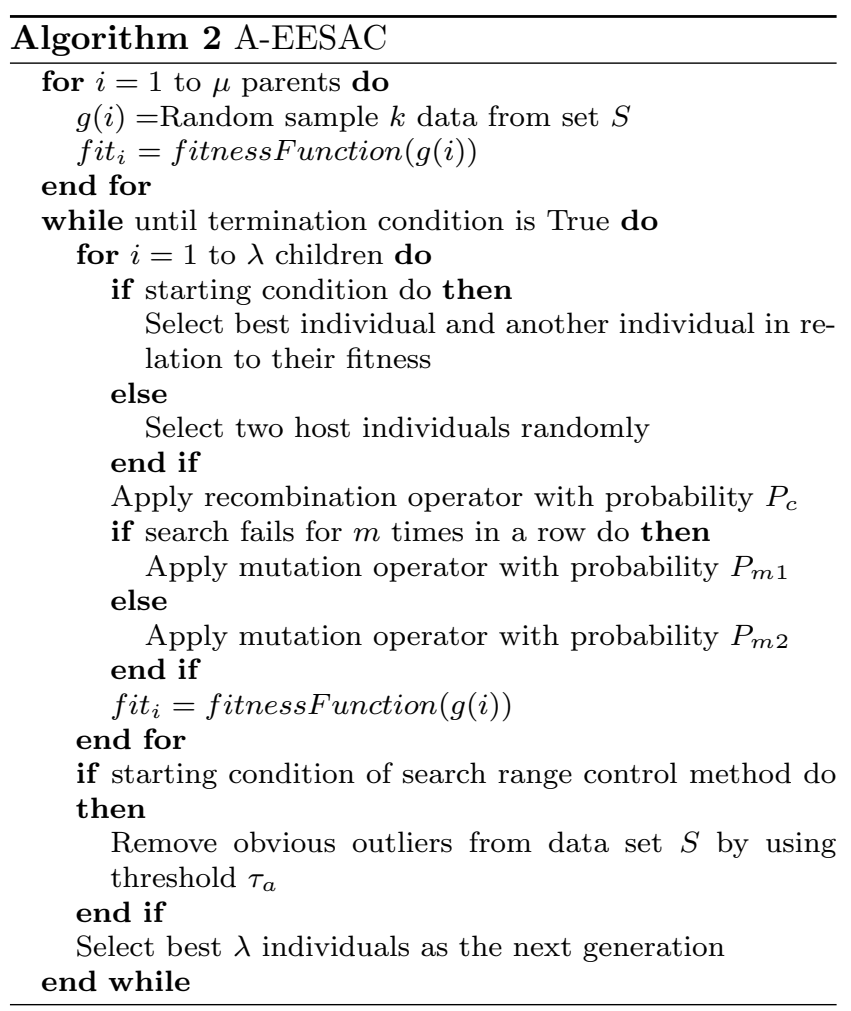

\section{Experimental result}

We conducted an experiment on $3 \mathrm{D}$ reconstruction in order to verifying the effectiveness of A-ESSAC in a moving image. Left and right images were acquired by 
using a robot arm equipped with two cameras and the number of frames is 670 (Fig.5). Figure 6 shows examples of camera images from the left camera. Table 1 shows the experimental parameters of A-ESSAC, and these parameters were determined empirically.

Figure 7 shows the experimental result of homography matrix estimation between $D_{L}(t)$ and $D_{R}(t)$ and Table 3 shows the results of a comparison between AESSAC, RANSAC and GASAC with Simulated Annealing (GASAC-SA) [19] (The number of trials of each dataset is set to 1000). A-ESSAC and GASAC-SA outperforms RANSAC in all of the datasets from the viewpoint of the average fitness value since the search capability of the genetic operators. In addition, A-ESSAC performs the search range control for searching better feasible solution by removing the obvious outliers from the dataset. Therefore, the result of A-ESSAC is slightly better than that of GASAC-SA. Furthermore, the computational time of A-ESSAC is less than the other methods because of the search range control. From the result, A-ESSAC can improve the trade-off between computational cost and stability of search.

Next, Table 3 and Fig. 8 show a result of a comparison between A-ESSAC using the adaptive and selfadaptive mutations (Eq.(20) and Eq.(21)), A-ESSAC using only the adaptive mutation (Eq.(20)) and ESSAC using the fixed mutation rate in [6] (The mutation rate $\left.P_{m}=0.125\right)$ for verifying the effectiveness of our proposed mutation strategy. In this experiment, the number of trials is set to 1000 . From the result, our proposed mutation strategy outperforms the other mutation strategies in the viewpoint of the average fitness value and variance. Furthermore, Fig.9 shows a result of the mutation rate transition for considering the result of Table 3. In Fig.9, the self-adaptive mutation was performed in the 5 th and 13 th generations since AESSAC could not improve the fitness value for 5 times in a row. After these self-adaptations, the search strategy is similar to the random search method since the mutation rate was over 0.4 for increasing the genetic diversity. In the 47th generation, the best fitness value was improved, and the mutation rate was decreased for searching the better combination in the current gene set. In this situation, the crossover operation is dominant compared with the mutation operator since the mutation rate is less than 0.1 . From these results, by changing the search strategy according to the best fitness value, A-ESSAC could search the feasible combination in this result (Fig.9). Although A-ESSAC sometimes gets stuck in a local optimum in some trials (e.g. The variance results ( $t=1$ and 230) of the fitness value are slightly large), A-ESSAC can recover from such a situation by extracting the correct pairs between the next images. Therefore, the result of $3 \mathrm{D}$ reconstruction could be stably performed by using A-ESSAC (Fig.10).

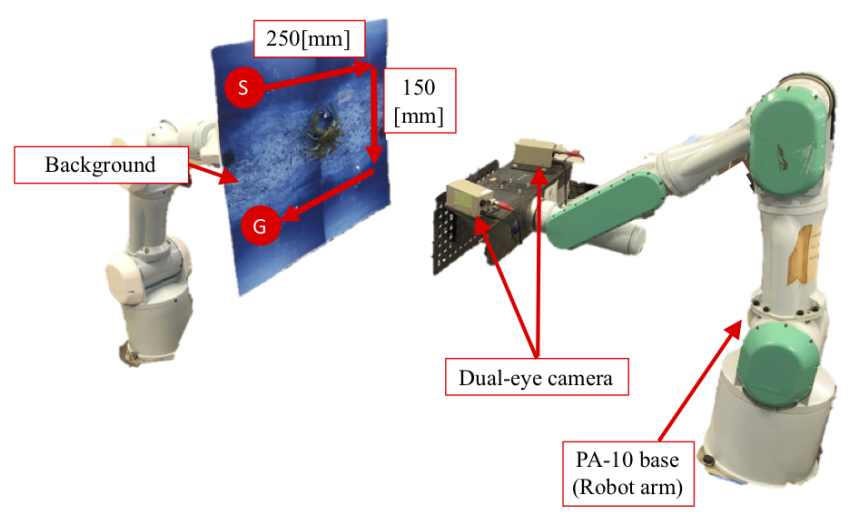

Fig. 5 Experimental environment ( $\mathrm{S}$ and $\mathrm{G}$ indicate Start and Goal, respectively).

Table 1 Experimental condition and Parameter setting

\begin{tabular}{l|c}
\hline The number of evaluations & 1000 \\
\hline The number of trials & 10 \\
\hline The number of parents $\mu$ & 100 \\
\hline The number of offspring $\lambda$ & 10 \\
\hline Threshold of Inlier/Outlier $\tau$ & 3 \\
\hline Threshold of search range control $\tau_{a}$ & 10 \\
\hline Initial condition of search range control $\alpha$ & 0.01 \\
\hline
\end{tabular}

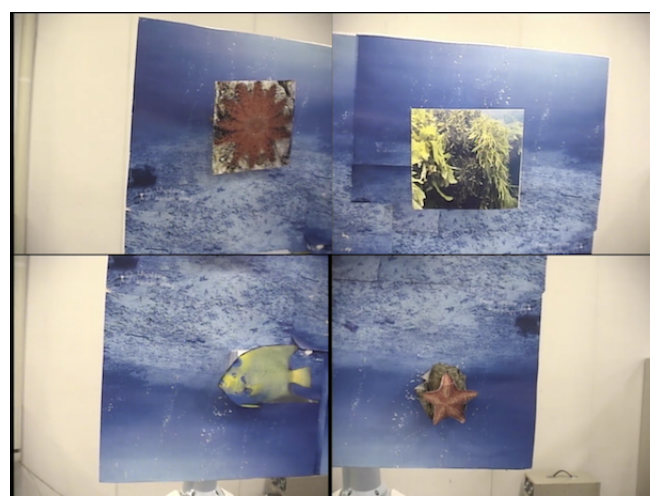

Fig. 6 Examples of camera images from the left camera.

\section{Conclusion}

In this paper, we applied A-ESSAC to 3D reconstruction method using two cameras. At first, we explained 


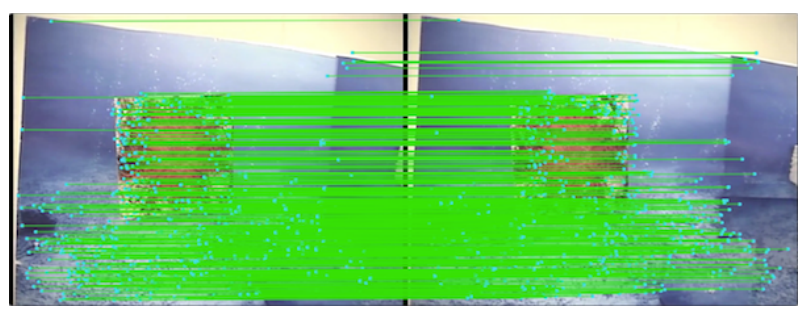

(a) $\mathrm{t}=1$

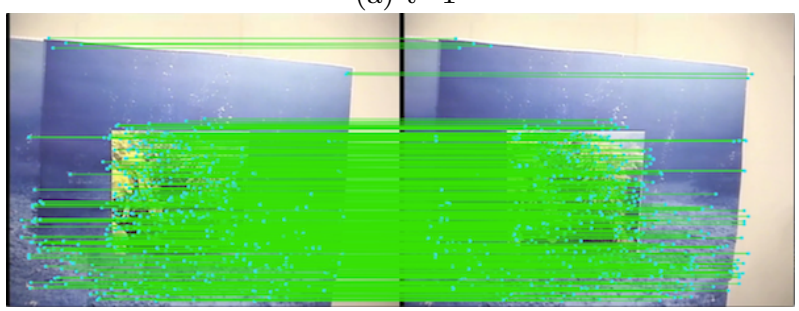

(b) $\mathrm{t}=230$

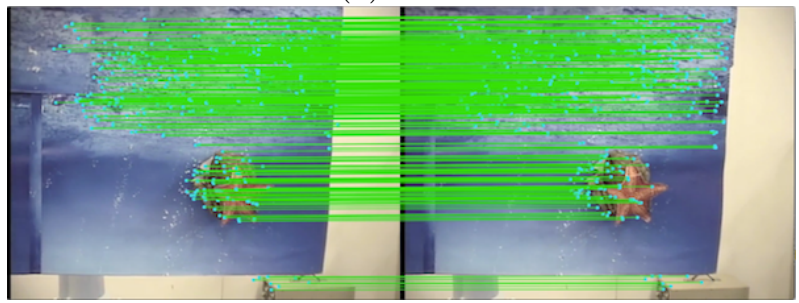

(c) $\mathrm{t}=380$

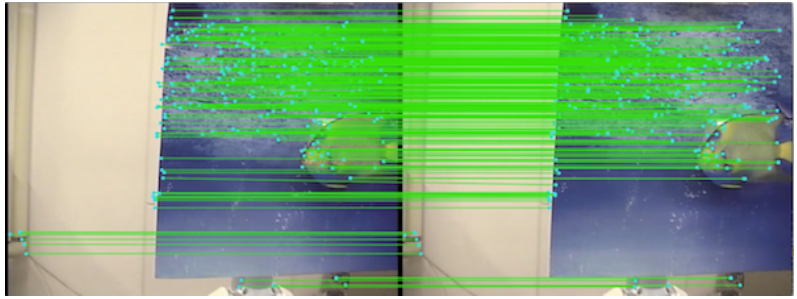

(d) $\mathrm{t}=670$

Fig. 7 Experimental results of a homography matrix estimation (Left and Right images are the measurement data from the left and right camera, respectively).

Table 2 Results of a comparison experiment between AESSAC, RANSAC and GASAC-SA

\begin{tabular}{c|c|c|c}
\hline & \multicolumn{4}{|c}{ Average fitness value (Variance) } \\
\hline Step(t) & A-ESSAC & RANSAC & GASAC-SA \\
\hline 1 & $168.7(1243)$ & $99.2(2624)$ & $150.8(664)$ \\
\hline 230 & $147.2(900)$ & $58.7(1619)$ & $143.4(316)$ \\
\hline 380 & $143.7(41)$ & $113.5(660)$ & $139.0(286)$ \\
\hline 670 & $239.6(50)$ & $196.7(1199)$ & $218.6(234)$ \\
\hline \multicolumn{4}{|c}{ Average computational time (Variance) } \\
\hline 1 & $25.8(47)$ & $52.4(2)$ & $76.2(11)$ \\
\hline 230 & $32.6(197)$ & $50.8(2)$ & $62.9(12)$ \\
\hline 380 & $17.8(3)$ & $30.2(1)$ & $45.5(4)$ \\
\hline 670 & $26.1(5)$ & $44.5(3)$ & $48.9 .5(5)$ \\
\hline
\end{tabular}

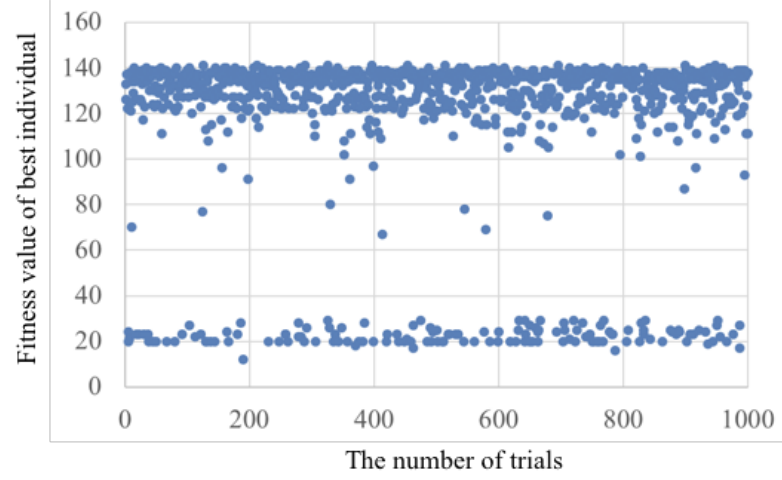

(a) A-ESSAC

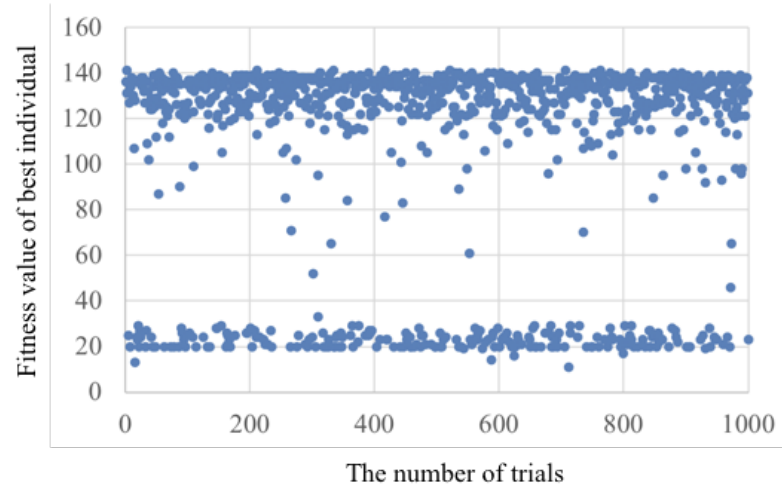

(b) Eq. (20)

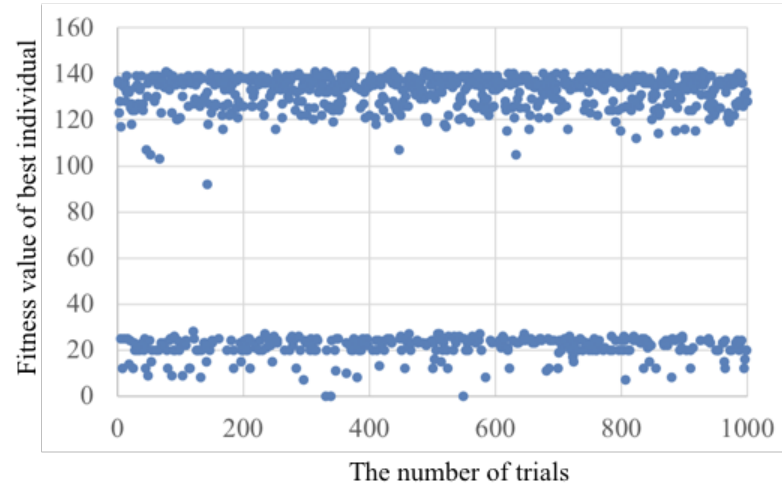

(c) $[6]$

Fig. 81000 trials Result of the comparison experiment shown in Table 3

Table 3 A Result of a comparison experiment between AESSAC (using Eq (20) and (21)), A-ESSAC (using only Eq (20)) and fixed value in [6]

\begin{tabular}{c|c|c|c}
\hline & \multicolumn{3}{|c}{ Average fitness value (Variance) } \\
\hline Step(t) & A-ESSAC & Eq. (20) & {$[6]$} \\
\hline 35 & $115.3(1497)$ & $106.2(2050)$ & $96.3(2765)$ \\
\hline
\end{tabular}




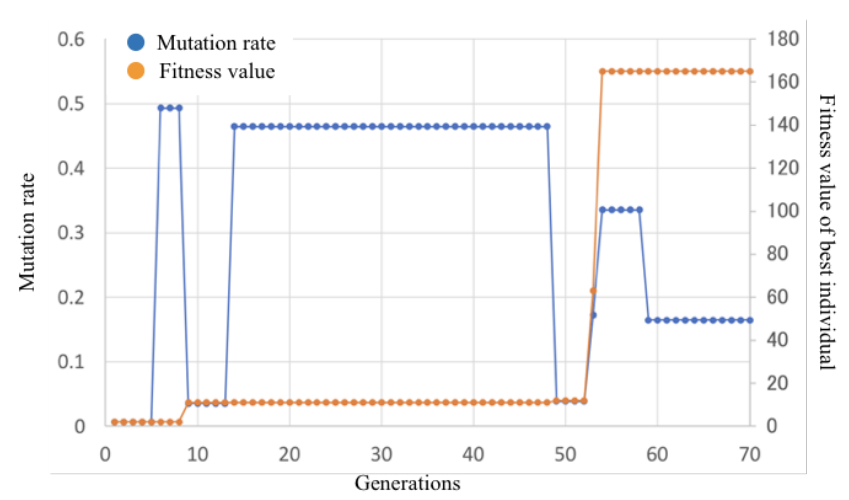

Fig. 9 An example of mutation rate transition $(t=1)$. Blue and Orange dots indicate the result of the mutation rate and fitness value of best individual respectively.

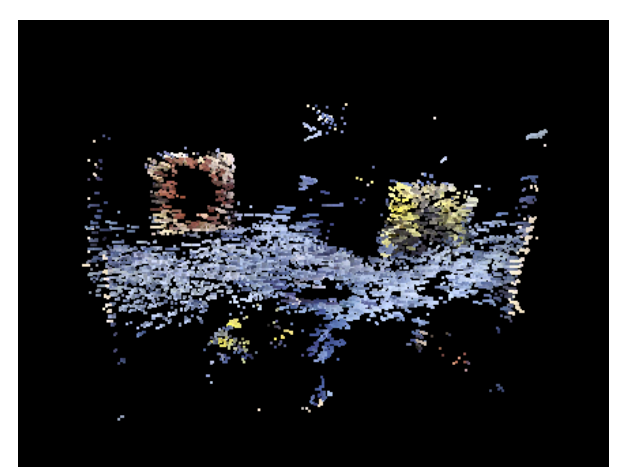

(a) Front view

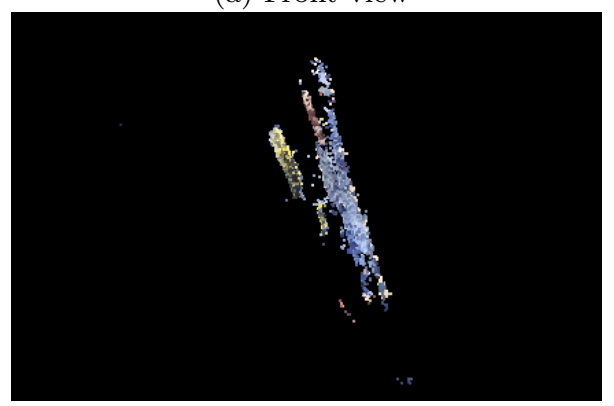

(b) Side view

Fig. 10 Experimental result of 3D reconstruction using two camera images.

the $3 \mathrm{D}$ reconstruction method from two cameras and defined the homography matrix estimation problem. Next, we explained A-ESSAC whose search strategy is based on Evolution Strategy in order to maintain the genetic diversity. In the experiments, we showed that A-ESSAC outperforms RANSAC in the average fitness and computational time and our proposed method could reconstruct the 3D model from two cameras. However, our proposed method has the problem of the accuracy of $3 \mathrm{D}$ reconstruction because of accumulated errors in each frame. Therefore, we will apply a closed- loop method to our proposed method for improving the accuracy of the 3D model.

\section{References}

1. Fischler M, Bolles R (1981), Random Sample Consensus: A Paradigm for Model Fitting with Applications to Image Analysis and Automated Cartography, Communications of the ACM, 24(6), pp. 381395

2. Adam A, Chatzilari E, Nikolopoulos S, and Kompatsiaris I, (2018), H-RANSAC: A hybrid point cloud segmentation combining 2D and 3D data, ISPRS Ann, Photogramm, Remote Sens. Spat. Inf. Sci, 4(2), pp. 1-8

3. Narksri P, Takeuchi E, Ninomiya Y, Morales Y, Akai N, and Kawaguchi N, (2018), A slope-robust cascaded ground segmentation in 3D point cloud for autonomous vehicles, In 2018 21st International Conference on Intelligent Transportation Systems (ITSC), pp. 497-504

4. Qian X, Ye C (2014), NCC-RANSAC: A Fast Plane Extraction Method for 3-D Range Data Segmentation, Cybernetics, IEEE Transactions on, 44(12), pp.2771-2783

5. Choi S, Taemin K, Wonpil Y (2009), Performance evaluation of RANSAC family, Journal of Computer Vision, .24(3) pp.1-12

6. Rodehorst V, Hellwich O (2006), Genetic Algorithm SAmple Consensus (GASAC) - A Parallel Strategy for Robust Parameter Estimation, In Proceedings of the IEEE Conference on Computer Vision and Pattern Recognition Workshop, pp.103-110

7. Shojaedini E, Majd M, Safabakhsh R (2019), Novel adaptive genetic algorithm sample consensus, Applied Soft Computing, vol. 77, pp. 635-642.

8. Toda Y, Kubota N (2016), Evolution Strategy Sampling Consensus for Robust Estimator, J. Adv. Comput. Intell. Intell. Inform., 20(5), pp. 788-802

9. Lowe D. G (1999), Object recognition from local scaleinvariant features, Proc. of IEEE International Conference on Computer Vision, pp.1150-1157

10. Bay H, Tuytelaars T, Gool L. V (2006), Surf: Speeded up robust features, in European Conference on Computer Vision, pp. 404-417

11. Cornelis N, Leibe B, Cornelis K, L. Gool V (2008), 3D Urban Scene Modeling Integrating Recognition and Reconstruction, International Journal of Computer Vision, 78 (23), pp. 121-141

12. Cyganek B, Siebert J. P (2009), Introduction to 3D Computer Vision Techniques and Algorithms, Wiley, John \& Sons, Incorporated

13. Torr P.H.S (2002), Bayesian model estimation and selection for epipolar geometry and generic manifold fitting, International Journal of Computer Vision, 50(1), pp. 3561

14. Chum O, Matas J (2005), Matching with PROSAC - Progressive Sample Consensus, In Conference on Computer Vision and Pattern Recognition, pp. 220226

15. Sharp G, Lee S, Wehe D (2002), ICP registration using invariant features, IEEE Transactions on Pattern Analysis and Machine Intelligence, 24(1), pp.90-102

16. Horn B (1987), Closed-Form Solution of Absolute Orientation Using Unit Quaternions, Journal of the Optical Society of America A, 4(4), pp.629642

17. Fogel D.B (1995), Evolutionary Computation, IEEE Press

18. Rechenberg I (1973), Evolutionsstrategie: Optimierung technischer systeme nach prinzipien der biologischen evolution, Stuttgart: FrommannHolzboog Verlag 
19. Vasconcelos F, Henggeler C, and Barreto J. P (2011), Adaptive and Hybrid Genetic Approaches for Estimating the Camera Motion from Image Point Correspondences, ACM Conf. in Genetic and Evolutionary Computing Conference, pp. 949-956 